\title{
SOBRE EL MITO DEL SÍNDROME DE ALIENACIÓN PARENTAL (SAP) Y EL DSM-5
}

\section{ON THE MYTH OF PARENTAL ALIENATION SYNDROME (PAS) AND THE DSM-5}

\author{
Ramón Vilalta' y Maxime Winberg Nodal ${ }^{2}$ \\ 'Instituto de Medicina Legal y Ciencias Forenses de los Juzgados de Oviedo. ${ }^{2}$ Instituto de Medicina Legal y \\ Ciencias Forenses de los Juzgados de Toledo
}

El presente artículo aborda la controversia surgida sobre la existencia del Síndrome de Alienación Parental (SAP) o cualquiera de sus denominaciones alternativas. Las críticas al SAP comprenden argumentos de diferente naturaleza: desde las críticas sobre la personalidad del creador del término, hasta la más repetida de que el SAP no consta en el Manual Diagnóstico y Estadístico de los Trastornos Mentales DSM-5. En este artículo se analizan algunas de esas crificas en el contexto judicial español, en particular por su relevancia, las expuestas en la Guía práctica del Grupo de Expertas y Expertos en violencia doméstica y de género del Consejo General del Poder Judicial (CGPJ), publicada en 2016; y se argumenta cómo el SAP sí que es ampliamente reconocido por la comunidad cientifica y profesional, y puede ser descrito y clasificado en el DSM-5 como un "Problema de relación entre padres e hijos V61.20 (Z62.820)".

Palabras clave: Síndrome de Alienación Parental, DSM, Divorcio, Niños, Distanciamiento afectivo.

This paper discusses the controversy over the existence of the Parental Alienation Syndrome (PAS) or any other name it may be given. The negative judgements over PAS are different in nature: from criticism about the personality of the term's creator, to the most repeated one that says PAS does not exist in the Diagnostic and Statistical Manual of Mental Disorders DSM-5. This paper analyses some of these criticisms in the Spanish judicial context, especially those exposed in the Guidelines of the Group of Experts on the Fight against Domestic and Gender Violence of the General Council of the Judiciary (CGPJ), published in 2016; and shows how the PAS is widely recognized by the professional and scientific community and it may be described and classified in the DSM-5 as a "Parent-Child Relational Problem V61.20 (Z62.820)".

Key words: Parental Alienation Syndrome, DSM, Divorce, Children, Estrangement.

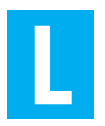

os Magistrados del Grupo de Expertas y Expertos en violencia doméstica y de género del Consejo General del Poder Judicial (CGPJ), en reunión celebrada el día 13 de octubre de 2016 aprobaron la Guía práctica de la Ley Orgánica 1/2004, de 28 de diciembre, de Medidas de Protección Integral contra la Violencia de Género (Carmona et al., 2016). En el capítulo X de la misma, bajo el título: Otros aspectos a tener en cuenta en la Actividad Jurisdiccional, incluyen cuatro páginas de comentarios acerca del llamado Síndrome de Alienación Parental (SAP). Sometemos a análisis el apartado $X$ de esta Guía pues -en su brevedad- pretende dictar doctrina en el ámbito forense y puede servir a nuestro análisis del mito sobre la inexistencia del fenómeno SAP, basado -entre otros argumentos- en su falta de inclusión en las clasificaciones diagnósticas, cuestión concreta sobre la que se centra este artículo. El problema resulta de gran interés para el trabajo de todos los actores jurídicos afectados por estas cuestiones, dado el prestigio del

Recibido: 21 abril 2017 - Aceptado: 22 mayo 2017

Correspondencia: Maxime Winberg Nodal. Instituto de Medicina

Legal y Ciencias Forenses de los Juzgados de Toledo. C/ Marqués de Mendigorría s/n. 45007 Toledo. España.

E-mail:maximo.winberg@justicia.es
CGPJ y las posibles implicaciones de esta Guía a nivel de práctica jurídica y forense.

\section{DEFINICIÓN DEL SAP}

Gardner (1985) acuñó el posteriormente controvertido término Síndrome de Alienación Parental (SAP), que aquí consideraremos equivalente al más empleado actualmente de Alienación Parental (AP), enmarcándolo principalmente dentro del contexto de un divorcio contencioso. Lo típico del SAP es que el hijo rechace y critique reiteradamente a uno de sus progenitores. Tales críticas son injustificadas o claramente exageradas. El menor habla de ese progenitor "odiado" en términos despectivos, sin avergonzarse ni sentir culpa por hacerlo. A veces su discurso brota a la primera pregunta de alguien relacionado con el conflicto (letrados, jueces, profesionales de salud mental, etc.) y adquiere la apariencia de "una letanía". En ocasiones incluso puede observarse que el discurso y el léxico del menor es muy similar al que usa el otro progenitor, al que afirma sentirse unido en exclusividad. Este rechazo es un proceso complejo, en el que juegan su papel ambos progenitores y el propio hijo. En el SAP un progenitor modela o programa al hijo para que rechace al otro. Además de los mensajes procedentes del lavado de cerebro 
del adulto, el niño debe realizar aportaciones propias al rechazo, dando cuenta de haber adquirido independencia en su discurso y conducta para mantenerlo. Evidentemente, durante la evaluación debe descartarse la existencia de un maltrato real, que haría incompatible la determinación de un SAP (Gardner, 1992).

Esta situación fue tratada con otros vocablos y matices por muchos otros autores antes y después de Gardner. A ello se refería la descripción del «progenitor programador» de Duncan (1978), o a lo que Wallerstein y Kelly (1980) llamaron "Síndrome de Medea», terminología que también adoptó Jacobs (1988). También se han propuesto otros términos que aluden parcialmente al mismo concepto, como: el «síndrome SAID» (Sexual Allegations In Divorce) de Blush y Ross (1987); el término "parentectomy» de Williams (1990); el «síndrome de la madre maliciosa» de Turkat (1995); la "alienación parental» de Darnall (1999); o la reformulación del «niño alienado» de Kelly y Johnston (2001). En España, Granados (1987) definió algunas características de estas situaciones de alta conflictividad destacando los aspectos irracionales de tales conflictos familiares. Posteriormente, Ramírez, de Luis e Ibáñez (1994) se refirieron a estas situaciones comparándolas con el "síndrome de Estocolmo». Durante los últimos años, el estudio del término SAP se ha extendido en nuestro país (Arce, Fariña y Seijo, 2005; Aguilar, 2004, 2014; Bolaños, 2002; Junco, Nieves y Fernández, 2014; Luengo y Coca, 2007; Muñoz, 2011; Segura, Gil y Sepúlveda, 2006; Tejedor, 2006; Vallejo, Sánchez-Barranco y Sánchez-Barranco, 2004; Vilalta, 2011).

Pese a tal variedad de trabajos y terminología sobre el SAP, la referida Guía pretende censurar su uso en cualquiera que sea su denominación, a saber: "La utilización del llamado "Síndrome de Alienación Parental" (en adelante, SAP), o la de una denominación alternativa pero con la misma virtualidad..." (Carmona et al., 2016, p. 271). Esta premisa imposibilitaría cualquier análisis del problema y entraría dentro de la corriente que Carbó (2011) denomina negacionismo fanatizado del $S A P$, emulando controversias de tinte nominalista que ya fueron bien abordadas por otros (Arch, Molina y Jarné, 2008; Chacón, 2008), y que centrarían el estudio del asunto en términos filosóficos y no como un problema de conducta de índole psicológica.

Independientemente del debate nominalista, si en el contexto forense el bien jurídico superior está en proteger a los menores de cualesquiera malos tratos, resulta necesario describir las conductas y manifestaciones que definen cada caso concreto. Es ésta la cuidada tarea que se lleva a cabo por parte de los operadores de la Administración de Justicia, sin entrar en discusiones nosológicas, y dedicándose con cautela a comprobar que cuando un rechazo se dé, no suceda por haber sufrido ese menor una situación de malos tratos, lo que justificaría su protección inmediata.

\section{CRÍTICAS CONTRA LA EXISTENCIA DEL SAP}

Las críticas al SAP que vamos a examinar siguiendo el capítulo X de la Guía Práctica (Carmona et al., 2016), se pueden agrupar en tres diferentes argumentaciones: las que incluyen descalificaciones a la personalidad de Gardner y a la orientación de su trabajo; las críticas de corte clínico y sobre el consenso científico al respecto del constructo SAP; $y$, por último, las que pretenden fundamentarse en contenidos jurisprudenciales.

\section{CRÍTICAS "AD HOMINEN"}

El citado Grupo de expertos del CGPJ mantiene que la Ley lntegral 1/2004 de Medidas de Protección Integral contra la Violencia de Género ha supuesto la aparición en escena de reacciones para su minimización, considerando la utilización del llamado "Síndrome de Alienación Parental" una de estas reacciones. Se refieren al SAP como un recurso que habría sido creado por Richard Gardner (1985), un psiquiatra al que a pie de página (citando una sentencia de la Sección $6^{a}$ de la Audiencia Provincial de Vizcaya de 27-3-2008), se le imputa pedofilia (Carmona et al., 2016). Sorprende que no se le tache también de adivino, pues se advierte que el psiquiatra habría formulado su recurso en Estados Unidos, unos 19 años antes de que en España se publicase dicha Ley Integral. Emplear la falacia ad hominen para criticar la obra de alguien es un recurso falto de sutileza, pues quien lo emplea no parece tener a su alcance otra argumentación sustantiva.

Tal falacia parece ser un recurso frecuente. El texto del Grupo de Expertos cita a pie de página al Dr. Fink, ex Presidente de la Asociación Psiquiátrica Americana, quien declaró en marzo de 2010, que los "grupos de defensa de los derechos de los padres" habían pedido al grupo de trabajo del DSM que incluyeran el SAP porque no les gustaba ser molestados cuando estaban abusando de sus hijos (Fink 2010). Sin embargo, en mayo se retractó en la misma publicación:

"Pido disculpas por sugerir que los padres que acusan a las madres de SAP están abusando sexualmente de sus hijos. Fue claramente un exceso del que me retracto... Yo no niego que la alienación parental suceda y que un montón de gente resulta dañada cuando existe un alienador" (citado en Lorandos, Bernet y Sauber, 2013, p. 494).

Sin embargo, procede señalar aquí uno de los aciertos conceptuales del texto del citado Grupo de Expertos, que también serviría para desligar definitivamente este supuesto síndrome del objeto de la Ley contra la Violencia de Género, cuando -en contradicción con lo previo- reconoce que incluso Gardner excluía la aplicación de su teoría en los casos en que se evidenciaba una situación de violencia, abuso o negligencia. Efectivamente, Gardner $(1985,1992)$ señala desde su introducción, que el término solo es aplicable cuando el progenitor rechazado no ha mostrado conductas que justifi- 
quen dicho rechazo filial, dejando claro que hablaba de otras situaciones con las que establecía una clara diferenciación.

De todos modos, el citado Grupo insiste en ligar el denominado SAP con la Ley de Violencia de Género, y alude al trabajo de Escudero, Aguilar, y de la Cruz (2008a, 2008b) en el que negaban la existencia del SAP como una patología que implicase tratamiento médico, algo que a la sazón resulta irrelevante en términos DSM-5 pues: "el diagnóstico de un trastorno mental no equivale a una necesidad de tratamiento" (Asociación Psiquiátrica Americana, 2014, p. 20); aunque sí salvaban la denominación del fenómeno como AP: "El presente trabajo se centra exclusivamente en la construcción del "síndrome de alienación parental» de Gardner o SAP. No alude por tanto al concepto de "alienación parental» con el cual puede confundirse, en gran medida por su similitud gramatical" (Escudero et al., 2008b, p. 286).

También se publicó en 2009 un libro desaconsejando emplear el diagnóstico SAP por pretendido (Váccaro y Barea, 2009), autoras que al final del mismo también reconocían ese tipo de manipulación infantil. Véase en su Apéndice 1-Algunas Consideraciones:

"Nada en este libro niega que en algunos divorcios controvertidos, los hijos no puedan ser manipuladas/os por uno o ambos progenitores. Por mi parte, sé que esto ocurre -aunque en menor medida de lo que se cree y supone- $y$ en general, este accionar termina haciendo crónico un litigio que parece tener como único objetivo desde la pareja, el "no separarse" nunca, aunque el vínculo que establezcan a partir de ese momento, sea el judicial y ya no esté en juego el amor sino el poder" (Váccaro y Barea, 2009).

Efectivamente, aunque el dinero sea el reforzador generalizado por excelencia, quizás en esta casuística lo sea el poder, a saber: "la posibilidad de disponer de las contingencias que afectan a las conductas de los demás" (Pérez, 2004).

El poder, reforzador generalizado de uno $\mathrm{u}$ otro progenitor $y$ la contingencia de su acción, es además una motivación cuya interpretación puede ser contemplada desde la Psicología normal sin recurso a la psicopatología. Estamos plenamente de acuerdo con dichas autoras cuando recomiendan un estudio individualizado de cada caso.

\section{CRÍTICAS PSEUDO-CLÍNICAS}

Entrando en el núcleo del debate, el mencionado Grupo de Expertos pretende emitir un argumento de autoridad que afecte a la práctica forense general, y lo hace mediante este otro tipo de falacia, que consiste concretamente en afirmar que tal Síndrome de Alienación Parental no figura en ninguna de las clasificaciones diagnósticas estadísticas internacionales sobre trastornos mentales y del comportamiento, y por lo tanto que pese a su resonancia no existe:
"No obstante, a pesar de la difusión y popularización de este pretendido síndrome en nuestro país, el SAP no ha sido reconocido por ninguna asociación profesional ni cientifica, habiendo sido rechazada su inclusión en los dos grandes sistemas diagnósticos de salud mental utilizados en todo el mundo, el DSM-V de la Asociación Americana de Psiquiatría, y el ICE-10 de la Organización Mundial de la Salud" (Carmona, et al., 2016, p. 272).

Un "síndrome" se definía por el DSM-IV-TR como: "una agrupación de signos y síntomas basada en su frecuente coocurrencia, que pueden sugerir una patogenia, una evolución, unos antecedentes familiares o una selección terapéutica comunes" (APA, 2002, p. 921). Admitimos el uso de tal término en múltiples ocasiones, sin ir más lejos en la propia Ley Integral 1/2004, cuando en el apartado primero de su Exposición de Motivos hace mención a otro:

"Existe ya incluso una definición técnica del síndrome de la mujer maltratada que consiste en "las agresiones sufridas por la mujer como consecuencia de los condicionantes socioculturales que actúan sobre el género masculino y femenino, situándola en una posición de subordinación al hombre y manifestadas en los tres ámbitos básicos de relación de la persona: maltrato en el seno de las relaciones de pareja, agresión sexual en la vida social y acoso en el medio laboral" (Ley Integral 1/2004, p. 42166).

Todos estaríamos de acuerdo en el despropósito que sería pretender negar la existencia de mujeres maltratadas por el mero hecho de que tal síndrome no apareciera en una clasificación médica.

En realidad, la nueva versión del DSM-5 deja de distinguir entre trastornos y síndromes, $y$, en cualquiera de sus versiones, es básicamente una convención de los especialistas sobre el estado de la cuestión en un momento determinado, pues sus criterios son variables en el tiempo.

Así, el sistema DSM incluía la homosexualidad entre sus trastornos hasta el año 1973, y hubo de esperarse 17 años más para que la Organización Mundial de la Salud (OMS) la excluyera de la Clasificación Estadística Internacional de Enfermedades y otros Problemas de Salud (Colegio Oficial de Psicólogos COP, 2017). Por otra parte, distintos problemas que no figuran entre los trastornos incluidos en estas clasificaciones, también ocupan a investigadores y merecen la atención de los profesionales de salud mental, sin que por ello tenga que considerarse que el profesional deba evitar describirlos. Pongamos un novísimo ejemplo, la "adicción a Internet", bajo cuya denominación se convocan congresos y jornadas, se escriben artículos científicos y se dispensa atención clínica, sin que nadie se preocupe de elaborar documentos ni peticiones públicas para que se suspendan tales estudios, evaluaciones o tratamientos psicológicos por no constar aún en los manuales diagnósticos y estadísticos DSM o 
CIE. Véase por último, cómo el DSM-5 sigue manteniendo como trastorno la «disforia de género» que antes figuraba clasificada como F64.x "Trastorno de la identidad sexual» (DSM-IV-TR, 2002) patologizando aún a personas transgénero y transexuales.

En conclusión, todos los manuales de clasificación incluyeron trastornos que más tarde eliminaron; también existen trastornos que actualmente no constan pero que probablemente figuren más adelante; y quizás mantienen otros que luego no lo serán. Por lo tanto, no se puede considerar que la inclusión o no de un fenómeno relacional y conductual complejo en estas clasificaciones, permita concluir fehacientemente sobre su existencia.

Por otra parte, el Grupo de Expertos y Expertas (Carmona et al., 2016) también apoya su argumentación en una declaración de 1996 del Grupo de Trabajo sobre "Violencia y Familia" de la Asociación Americana de Psicología (APA), que pretenden hacer pasar como su postura oficial. Sin embargo, omiten citar la nota de prensa de 1 de enero de 2008, en la que la APA afirmaba que no tiene posición oficial sobre tal pretendido síndrome, y en la que, como es lógico, apela a los profesionales a tomar seriamente cualquier denuncia de violencia dentro de la familia (APA, 2008).

Podría argumentarse por el contrario, que varias asociaciones incluyen este problema del SAP como propio del estudio psicológico forense (Asociación de Psicólogos Forenses de la Administración de Justicia, 2016; Asociación lberoamericana de Psicología Jurídica y Forense, 2016), y que aparece en distintos manuales de psicología (Puckering 2010; Venzke 2010). También podemos señalar la extensa bibliografía que figura en los artículos de Bernet, Von Boch-Galhau, Baker y Morrison (2010) y de Bernet y Baker (2013).

Comienzan también a aparecer estudios meta-analíticos en los que se reconoce la creciente cantidad de trabajos al respecto del fenómeno y las limitaciones en el estatus empírico de la investigación en Alienación Parental (Mendes, Bucher-Maluschke, Vasconcelos, Fernandes y Costa, 2016; Saini, Johnston, Fidler y Bala, 2012). Estas limitaciones serían intrínsecas a la dificultad del estudio empírico de tales situaciones:

"Como señala el artículo de Saini, Jhonston, Fidler y Bala (2012), existen limitaciones metodológicas significativas en la investigación realizada sobre el SAP, pero también es difícil pensar en cómo obtener una muestra aleatoria de casos de alienación parental que cumpliera con tales exigencias. Las limitaciones de la investigación no cuestionan que existan investigaciones relevantes respecto al fenómeno, que van mucho más allá de observaciones informales o casos anecdóticos".

(Hynan 2015, p. 201)

Es decir, viene a ser otro Mito que exista tal consenso en el rechazo de las asociaciones profesionales o la comunidad científica hacia el fenómeno del SAP o de la AP, más bien pa- rece todo lo contrario. Incluso los más críticos vienen a reconocer en contradicción su existencia: "La mayor paradoja del $S A P$ es que ayuda a generar las condiciones de un segundo $S A P$, sólo que ahora inverso contra el progenitor diagnosticado como alienador y sobre el niño,..." (Escudero et al., 2008b, p. 307).

La situación planteada por los detractores del SAP con este argumento pseudo-clínico, recuerda a la disputa que mantuvieron los catedráticos jesuitas con Galileo sobre la existencia de las manchas solares, porque éstas no aparecían en la Biblia y suponían reconocerle un defecto a la "perfección" del sol. Carecía de interés para ellos mirar por un buen telescopio para verlas y extraer las lógicas consecuencias. Las manchas solares existieron para ellos, cuando finalmente la autoridad eclesiástica concluyó, independientemente de las observaciones de Galileo, que el sol podía tener defectos porque en la Biblia ya constaba que Josué había hecho parar el sol (un defecto) cuando solicitó a Yahvé que le dejara disponer de un horario más amplio para matar amorreos (Josué 10:13); y porque si Job obtuvo que el sol dejase de brillar (Job 9:7), sería también por alguna mancha (Beltrán Martí, 2005).

\section{CRÍTICAS DE ARGUMENTO PSEUDO-JURISPRUDENCIAL}

El Grupo de Expertos del CGPJ expone también en sus recomendaciones una línea argumental jurídica $\circ$ jurisprudencial. En primer lugar citan la ya mencionada Sentencia de la Sección $6^{\underline{a}}$ de la llma. Audiencia Provincial de Vizcaya, de 27 de marzo de 2.008; reiterando además los mismos argumentos de su anterior exposición de 2013 (Montalbán et al., 2013) que ya han sido comentados. Añaden referencia a dos Sentencias posteriores:

"En este sentido rechaza el citado síndrome la Sentencia 162/2016 del Tribunal Supremo de 16 de marzo de 2016, así como la Sentencia 399/2015 de la Sección $6^{a}$ de la Audiencia Provincial de Málaga de 30 de junio, cuando señala que "el denominado síndrome de alienación parental, conocido como el conjunto de síntomas que resultan del proceso por el cual un progenitor, mediante distintas estrategias, transforma la conciencia de sus hijos con objeto de impedir, obstaculizar o destruir sus vínculos con el otro progenitor, hasta hacerla contradictoria con lo que se esperaría de su condición, logrando provocar el progenitor alienador mediante un mensaje y un programa constituyendo lo que normalmente se denomina "lavado de cerebro", desarrollando los hijos que sufren este síndrome un odio patológico e injustificados hacia el progenitor alienado". Esta Sala comparte las profundas dudas científicas sobre la existencia de ese síndrome, y, en su caso, sus causas, consecuencias y soluciones, no obstante, sin entrar en dicho debate (...)" (Carmona et al., 2016, p. 274). 
Adviértase que la Sentencia que se transcribe no entra en el debate sobre la existencia o no del mencionado síndrome, pero es llamativo que la primera que citan tampoco lo haga, pues lo que consta allí es que los informes descartan que los niños de ese caso lo padezcan, sin entrar en su negación universal (Tribunal Supremo, 2016). No se presenta en realidad ninguna sentencia del Supremo que en su texto niegue explícitamente la existencia del SAP, algo además irrelevante en términos científicos. Por lo tanto, esta argumentación pseudo-jurisprudencial del Grupo de Expertos y Expertas, se vuelve autorreferencial (a su propio escrito de 2013) y yerma de contenido.

En sentido contrario, puede hacerse referencia a otras sentencias españolas y del Tribunal de Derechos Humanos de Estrasburgo, en las que se ha reconocido o rechazado la existencia de problemas de Alienación Parental o SAP en varios juicios concretos (Gaffal, 2012). De hecho, la propia Ley de Enjuiciamiento Civil española prevé medidas para solucionar situaciones de interferencia en las relaciones paternofiliales, a saber: "El incumplimiento reiterado de las obligaciones derivadas del régimen de visitas, tanto por parte del progenitor guardador como del no guardador, podrá dar lugar a la modificación por el Tribunal del régimen de guarda y visitas" (Ley de Enjuiciamiento Civil, Art. 766.3, 2000)

Resulta interesante también constatar la existencia de leyes que abordan específicamente el fenómeno de la alienación parental en otros países: como la Ley no 12.318 de 26 de agosto de 2010 de la República de Brasil; o la adición en 2014 del artículo 323 septimus, al Código Civil del Distrito Federal de México, en el que también se define y aborda tal problemática (Gaceta Oficial del Distrito Federal, 2014).

\section{EL SAP EN EL DSM-5}

Lo cierto es que ya Jarné y Arch (2009) señalaban la posible inclusión del SAP dentro de la categoría del DSM-IV denominada Z63.8 Problemas paterno-filiales [V61.20], lo que ponía en entredicho que los comportamientos que describe el SAP no encontrasen acomodo en dicha clasificación diagnóstica. Esta categoría que ellos señalaban debería usarse cuando el objeto de atención clínica fuese el patrón de interacción entre padres e hijos (p. ej., deterioro de la comunicación, sobreprotección, disciplina inadecuada) y estaría asociada a un deterioro clínicamente significativo de la actividad individual o familiar o a la aparición de síntomas clínicamente significativos en los padres o hijos, especificando Z63.1 si el objeto de atención clínica fuera el niño.

El grupo de trabajo de revisión del DSM-5 excluyó considerar la Alienación Parental como un trastorno mental, pero el Dr. Regier, vicepresidente del grupo de trabajo que redactaba el manual, consideró en una entrevista que se trataba de un problema relacional (Crary, 2012). La Asociación Americana de Psiquiatría editó finalmente la revisión de su Manual DSM-5
(2013) sin incluir el trastorno de alienación parental, pero realizando una notable actualización de la notación Z63.8 Problemas paterno-filiales [V61.20] dentro del epígrafe de: Otros problemas que pueden ser objeto de atención clínica y dentro un apartado que denomina: Problemas relacionados con la educación familiar, introduciendo el mismo código V61.20 (Z62.820): Problema de relación entre padres e hijos. Esta categoría V61.20, notablemente más detallada que en la anterior versión DSM-IV, permite diagnosticar lo que sigue (subrayado nuestro) y que parece corresponderse fielmente con lo que se conoce principalmente como SAP, a saber:

"En esta categoría el término "padre" se utiliza para referirse a cualquier cuidador principal del niño, ya sea un progenitor biológico, un padre adoptivo o de acogida, o cualquier otro familiar (como un abuelo) que desempeñe un papel parental para el niño. Esta categoría se debe utilizar cuando el principal objeto de atención clínica consiste en establecer la calidad de la relación padres e hijos o cuando la calidad de la relación padres e hijos está afectando al curso, pronóstico o tratamiento de un trastorno mental o médico. Habitualmente un "problema de la relación padres e hijos" va asociado a un deterioro funcional en los dominios conductuales, cognitivos o afectivos.

Son ejemplos de problemas conductuales: el inadecuado control, supervisión e implicación de los padres con el niño, la sobreprotección de los padres, la presión paterna excesiva, las discusiones que se agravan hasta llegar a la amenaza de violencia física y la evitación sin la resolución de los problemas. Los problemas cognitivos son atribuciones negativas a las intenciones de otros, hostilidad o convertir a otro en chivo expiatorio, y sensación de distanciamiento sin motivo. Los problemas afectivos pueden ser sensaciones de tristeza, apatía o rabia contra el otro miembro de una relación. Los clínicos han de tener en cuenta las necesidades de desarrollo del niño y su contexto cultural". (APA, 2014, p. 396).

Efectivamente, el SAP sólo es clasificable en el DSM-5 como un problema relacional o de interacción familiar desajustada, pues no es una enfermedad mental (Bernet y Baker, 2013; Lorandos et al., 2013; Siracusano, Barone, Lisi y Niolu, 2015).

La quinta edición del DSM describe los criterios diagnósticos que definen la existencia de un problema de relación entre progenitores e hijos de base psicológica, relacionado con la educación familiar; que causa un deterioro funcional definido en términos conductuales, en los tres posibles tipos de respuesta: conductual, cognitiva y emocional. Tiene valor que un manual de origen eminentemente psiquiátrico recurra a esta descripción funcional psicológica, lo que enmarca bastante adecuadamente la naturaleza del problema. Éste puede deberse a la existencia de una presión excesiva de algún cuidador 
principal para que el hijo o menor a su cuidado, haga atribuciones negativas a las intenciones de otro familiar (un rechazo injustificado) apareciendo un distanciamiento afectivo inmotivado y apatía o rabia contra el otro miembro de la relación.

La definición original de Gardner (1985) del SAP como una alteración que usualmente aparece en el contexto de un divorcio, en la que el niño desprecia y critica a uno de sus progenitores, cuando tal valoración negativa está injustificada o es exagerada, parece ajustarse a esta entrada, que resulta más concreta que la anterior al respecto del problema descrito con la notación V61.20.

Una palabra clave de la versión original en inglés del DSM-5 (APA, 2013) es el sustantivo estrangement, que ha sido traducido en la versión española como distanciamiento. Consultada la edición de 1989 de la Enciclopedia Webster, Diccionario Extenso del Idioma Inglés, el verbo estrange, raíz del sustantivo estrangement, se define como "cambiar los sentimientos o los afectos" y en segunda acepción "alienar los afectos". De hecho, la versión italiana del DSM-5 utiliza tal vocablo cuando traduce: "sentimenti non giustificati di alienazione" (APA, $2014 b$, p. 382). Sea distanciamiento sin motivo, destrucción de los afectos o alienación, la categorización del SAP puede ser efectuada en términos DSM-5 (APA, 2013) y, por lo tanto, parece otra falacia pretender utilizar tal argumento de autoridad para negar su existencia.

\section{CONCLUSIONES}

El capítulo X sobre el SAP de la Guía práctica aprobada por el CGPJ no incluye revisiones sistemáticas, meta-análisis, estudios empíricos, ni argumentación jurisprudencial o legislativa que sustente sus afirmaciones. Resulta curioso que en un contexto que debe ser exigente a la hora de la aceptación de pruebas científicas, los autores de una Guía práctica basen una consideración de importantes consecuencias de práctica jurídica, en argumentos tan débilmente sustentados.

El patrón relacional y contextual descrito bajo el concepto SAP, o cualquiera de las denominaciones alternativas previas o posteriores, no es ningún recurso creado para ocultar situaciones de malos tratos familiares contra las mujeres o los propios niños. De hecho, no se contempla su consideración en situaciones con constancia de tales malos tratos.

Las clasificaciones diagnóstico estadísticas de trastornos mentales y del comportamiento no agotan la descripción de las dinámicas conductuales y relacionales, sean éstas psicopatológicas o no. En realidad estas clasificaciones diagnósticas están en constante revisión, pues solo suponen cierto consenso ligado a un momento y contexto determinados. Apelar a ellas para negar o constatar un trastorno, un síndrome o una descripción conductual, relacional y contextual, tiene por lo tanto un valor muy relativo $y$, en todo caso, no es un argumento que deba ser utilizado para impedir la descripción de estas dinámicas si son detectadas en una valoración forense rigurosa.
El patrón relacional, contextual y conductual descrito bajo el concepto de SAP, o cualquiera de las denominaciones alternativas previas o posteriores, parece encontrar acomodo en la clasificación internacional de trastornos del DSM-5 descrito como un problema que puede ser objeto de atención clínica relacionado con la educación familiar. En concreto, dentro del código V61.20 (Z62.820) Problema de relación entre padres e hijos.

Tal vez debamos considerar si la descripción y el estudio de las características del llamado SAP, podrían haber logrado aportar al contexto judicial y psicológico-forense un marco psicodiagnóstico para la comprensión de tal fenómeno relacional; y si la comprensión de éste ha facilitado que menores envueltos en esas desajustadas dinámicas familiares hayan podido sustraerse a las mismas. Así parece desprenderse de una encuesta realizada en 2010 con ocasión de un encuentro de la Asociación de Juzgados de Familia y Conciliación cumplimentada por cerca de 300 asistentes, quienes en un $98 \%$ afirmaron creer que algunos niños son manipulados por un progenitor para rechazar irracional e injustificadamente al otro (Baker, Jaffee, Bernet y Johnston, 2011)

En la primera carta que Galileo le remite a Marco Velseri el 4 de mayo de 1612 sobre el mentado tema de las manchas solares, el genial astrónomo escribe: "Los nombres y los atributos de las cosas tienen que adaptarse a su esencia, y no la esencia a los nombres; porque primero existieron las cosas y después los nombres." (Panebianco, Gineprini y Seminara, 2011 , p. 3).

\section{CONFLICTO DE INTERESES}

No existe conflicto de intereses

\section{REFERENCIAS}

Aguilar, J. M. (2004). S.A.P., Síndrome de alienación parental: Hijos manipulados por un cónyuge para odiar a otro. Córdoba: Almuzara.

Aguilar, J. M. (2014). Síndrome de Alienación Parental. Madrid: Síntesis.

Arce, R., Fariña, F., y Seijo, D. (2005). Razonamientos judiciales en procesos de separación. Psicothema, 17, 57-63.

Arch, M., Molina, A., y Jarné, A. (2008). Aceptación y controversia del Síndrome de Alienación Parental. Revista del Collegi Oficial de Psicòlegs de Catalunya, 213, 30-33.

American Psychiatric Association (2002). DSM-IV-TR Manual Diagnóstico y estadístico de los trastornos mentales. Barcelona: Masson.

American Psychiatric Association (2013). DSM-5 Diagnostic and statistical manual of mental disorders. Washington: American Psychiatric Publication.

American Psychiatric Association (2014). DSM-5 Manual Diagnóstico y Estadístico de los Trastornos Mentales. Editorial Médica Panamericana. 
American Psychiatric Association (2014b). DSM-5. Manuale diagnostico e statistico dei disturbi mentali. Milán: Edizione Cortina Raffaello.

American Psychological Association (2008). Statement on Parental Alienation Syndrome. Recuperado de http://www.apa.org/news/press/releases/2008/01/passyndrome.aspx

Asociación Española de Psicólogos Forenses de la Administración de Justicia (2016). Comunicado de la APF ante las críticas a los psicólogos forenses públicos en relación con el SAP. Recuperado de https://www.psicologosforenses.org/comunicado-de-laapf-ante-las-criticas-a-los-psicologos-forenses-publicos-enrelacion-con-el-sap/\#more-837

Asociación Iberoamericana de Psicología Jurídica (2016). Declaración Asociación Iberoamericana de Psicología jurídica y forense -La vida en familia es un derecho humano. Lisboa, Portugal. Recuperado de http://www.familiaenderechos.es/2016/06/08/laasociacion-iberoamericana-de-psicologia-juridica-impulsanun-manifiesto-para-denunciar-la-alienacion-parental/

Baker, A., Jaffee, P., Bernet, W., y Johnston, J. (2011). Brief Report on Parental Alienation Survey. The Association of Family and Conciliation Courts eNEWS, 30 (2).

Beltrán Martí, A. (2005). Talento y poder. Pamplona: Editorial Laetoli.

Bernet, W., Von Boch-Galhau, W., Baker, A., y Morrison, S. (2010). Parental Alienation, DSM-V, and ICD-11. The American Journal of Family Therapy, 38, 76-187.

Bernet, W., y Baker, A. (2013). Parental Alienation, DSM-5, and ICD-11: Response to critics. The Journal of the American Academy of Psychiatry and the Law, 41, 98-104.

Bolaños, I. (2002). El Síndrome de Alienación Parental. Descripción y abordajes psico-legales. Psicopatología Clínica, Legal y Forense, 2, 25-45.

Blush, G. L., y Ross, K. L. (1987). Sexual allegations in divorce: The SAID syndrome. Family Court Review, 25(1), 1-11.

Carbó, E. (2011). Dos hipótesis sobre el negacionismo fanatizado del Síndrome de Alienación Parental. Recuperado de http://www.filo.cat/wpcontent/uploads/2013/04/Carb\%C3\%B3-Dos-hipotesisSAP-mayo-2011.pdf

Carmona, M. A., Llombart, C., Nadal, A., Gallego, G., Gómez, J.M., Magro, V., ... Lorente, M. (2016). Guía práctica de la Ley Orgánica 1/2004, de 28 de diciembre, de Medidas de Protección Integral contra la Violencia de Género. Madrid: Consejo General del Poder Judicial.

Chacón, F. (2008). El conceptualismo de Guillermo de Ockham y el debate sobre la existencia del Síndrome de Alienación Parental (SAP). Guía del Psicólogo, 284, 3.

Consejo Oficial de la Psicología (2017). Comunicado del COP sobre las "terapias de conversión" de la homosexualidad. Recuperado de http://www.infocop.es/view_article.asp?id=6660\&cat=9

Crary, D. (2012). Parental alienation not a mental disorder, American Psychiatric association says. Recuperado de $\mathrm{h}+\mathrm{t} \mathrm{p}:$ / / w w w. wash ing to n ti m e s.com / news/2012/sep/21/psychiatric-group-parental-alienationno-disorder/

Darnall, D. (1999). Parental alienation: Not in the best interest of the children. North Dakota Law Review, 75, 323-364.

Duncan, J.W. (1978). Medical, psychological and legal aspects of the child custody disputes. Mayo Clinic Proceedings, 53, 463-468.

Escudero, A.; Aguilar, L., y de la Cruz, J. (2008a). La construcción teórica del Síndrome de Alienación Parental de Gardner (SAP) como base para cambios judiciales de custodia de menores. Análisis sobre su soporte científico y riesgos de su aplicación. Recuperado de https://heterodoxia.files.wordpress.com/2009/08/sap-yfalacias.pdf

Escudro, A.; Aguilar, L., y de la Cruz, J. (2008b). La lógica del Síndrome de Alienación Parental de Gardner (SAP): «terapia de la amenaza». Revista de la Asociación Española de Neuropsiquiatría 28, 102, 283-305.

Fink, P.J. (2010a, March) Fink! Still at large. Clinical Psychiatry News, p. 6. Recuperado de http://www.mdedge.com/clinicalpsychiatrynews/article/2393 2 /pediatrics/fink-still-large-dsm-5-promises-changepractice/page/0/1

Gaceta Oficial del Distrito Federal (2014). Decreto por el que se adicionan Diversas Disposiciones al Código Civil para el Distrito Federal. Recuperado de http://www.ordenjuridico.gob.mx/Documentos/Estatal/Dis trito\%20Federal/wo95006.pdf.)

Gaffal, M. (2012). Parental alienation in divorce judgments. Revista para el Análisis del Derecho Indret, 4, 1-23. Recuperado de http://www.indret.com/pdf/929.en.pdf.pdf

Gardner, R. (1985). Recent trends in divorce and custody litigation. Academy Forum, 29(2), 3-7.

Gardner, R. (1992). The parental alienation syndrome, a guide for mental health and legal professionals. Cresskill, NJ: Creative Therapeutics.

Granados, F. (1987). Lo irracional en el conflicto familiar. Actualidad Civil, 35, 2087-2095.

Hynan, D. J. (2015). Child custody evaluation: New theoretical applications and Research. Springfield, IL: Charles C Thomas Publisher.

Jacobs, J.W. (1988). Euripides' Medea: A psychodynamic model of severe divorce pathology. American Journal of Psychotherapy, 42, 308-319.

Jarné, A., y Arch, M. (2009). DSM, salud mental y síndrome de alineación parental. Papeles del psicólogo, 30(1), 86-91. 
Junco, T., Nieves, V. E., y Fernández, P. F. (2014). Parental Alienation Gradient: Strategies for a syndrome. The American Journal of Family Therapy, 42(3), 217-231.

Kelly, J.B., y Johnston, J.R. (2001). The alienated child: A reformulation of parental alienation syndrome. Family Court Review, 39, 249-265.

Ley 1/2000 de Enjuiciamiento Civil. Boletín Oficial del Estado, Madrid, España, 8 de enero de 2000.

Ley $\mathrm{n}^{\circ} 12.318$ de 26 de agosto de 2010 de la República de Brasil. Recuperado de http://www.planalto.gov.br/ccivil_03/_ato2007-2010/2010/lei//12318.htm

Ley Orgánica 1/2004 de Medidas de Protección Integral contra la Violencia de Género. Boletín Oficial del Estado, Madrid, España, de 29 de diciembre de 2004.

Lorandos, D., Bernet, W., y Sauber, S. R. (2013). Parental alienation: The handbook for mental health and legal professionals. Springfield, IL: Charles Thomas Publisher.

Luengo, D., y Coca, A. (2007). Hijos manipulados tras la separación: cómo detectar y tratar la alienación parental. Barcelona: Viena Ediciones.

Mendes, J. A., Bucher-Maluschke, J. S., Vasconcelos, D. F., Fernandes, G. A., y Costa, P. V. (2016). Psycho-legal publications about parental alienation: an integrative review of literature in Portuguese. Psicologia em Estudo 21,161-174.

Montalbán, I., Bayo, J., Cueto, C., Gómez, J.M., Erice, E., Llop, P.,... Tardón, M. (2013). Guía de criterios de actuación judicial frente a la violencia de género (Actualización-2013), 166-169. Consejo General del Poder Judicial. Recuperado de http://www.violenciagenero.msssi.gob.es/profesionalesInvestigacion/juridico/protocolos/docs/GuiaActuacionjudicial2013.pdf

Muñoz Vicente, J.M. (2011). El constructo Síndrome de Alienación Parental (S.A.P) en Psicología Forense: Una propuesta de abordaje desde la evaluación pericial psicológica. Anuario de Psicología Jurídica, 20, 5-14.

Pérez, M. (2004). Contingencia y drama: La Psicología según el conductismo. Madrid: Minerva Ediciones.

Panebianco, B., Gineprini, M., y Seminara, S. (2011). La rivoluzione scientifica - La teoria copernicana. Recuperado de http://online.scuola.zanichelli.it/letterautori-files/volume-2/pdf-online/3-galilei_tema.pdf

Puckering, C. (2010). Parenting capacity and conduct. En J. M. Brown y E. A. Campbell (Ed.), The Cambridge Handbook of
Forensic Psychology (pp. 242-250). Cambridge: University Press.

Ramírez, M., de Luis, P., e lbáñez, V. (1994). Percepciones parentales en niños de familias separadas: ¿Una nueva versión del síndrome de Estocolmo? Anuario de Psicología Jurídica, 25-41.

Saini, M., Johnston, J. R., Fidler, B. J., y Bala. (2012). Empirical studies of alienation. En K. Kuehnle y L. Drozd (Eds.), Parenting plan evaluations: Applied research for the family court (pp. 399-441). New York: Oxford University Press.

Segura, C., Gil, M.J., y Sepúlveda, M.A. (2006). El Síndrome de Alienación Parental: una forma de maltrato infantil. Cuadernos de Medicina Forense, 43-44, 117-128.

Siracusano, A., Barone, Y., Lisi, G., y Niolu, C. (2015). Parental alienation syndrome or alienating parental relational behaviour disorder: a critical overview. Journal of Psychopathology, 21, 231-238.

Tejedor, A. (2006). El síndrome de alienación parental: una forma de maltrato. Madrid: Instituto de Orientación Psicológica.

Tribunal Supremo (2016). Sentencia no 162/2016 de TS, Sala 1ª de lo Civil, 16 de Marzo de 2016. Recuperado en http://supremo.vlex.es/vid/632399177

Turkat, I. (1995). Divorce related malicious mother syndrome. Journal of Family Violence, 10, 253-264.

Váccaro, S., y Barea, C. (2009). El pretendido Síndrome de Alienación Parental. Madrid: Desclée de Brower.

Vallejo, R., Sánchez-Barranco, F., y Sánchez-Barranco, P. (2004). Separación o divorcio: trastornos psicológicos en los padres y los hijos. Revista de la Asociación Española de Neuropsiquiatría, 92, 91-110.

Venzke (2010). Parental Alienation Syndrome. En Weiner y Craighead (Ed.) .Corsini Encyclopedia of Psychology (pp. 1157-1159). New Jersey: John Wiley and Sons.

Vilalta, R. J. (2011). Descripción del Síndrome de Alienación Parental en una muestra forense. Psicothema, 23(4), 636641.

Wallerstein, J.S. y Kelly, J.B. (1980). Surviving the break up: How children and parents cope with divorce. New York: Basic Books.

Williams, F. (1990). Preventing parentectomy after divorce. Fifth Annual Conference National Council for Children's Rights, Washington D.C. 